\title{
Metastasis-associated protein 1 promotes tumor invasion by downregulation of E-cadherin
}

\author{
WENHAO WENG $^{1 *}$, JIAYI YIN ${ }^{2 *}$, YUE ZHANG ${ }^{1}$, JIN QIU $^{3}$ and XINGHE WANG ${ }^{4}$ \\ ${ }^{1}$ Department of Laboratory Medicine, Shanghai Tenth People's Hospital, Tongji University School of Medicine, \\ Shanghai 200072; ${ }^{2}$ Department of Clinical Medicine, Shanghai Jiaotong University Affiliated Renji Hospital, \\ Shanghai 200127; ${ }^{3}$ Department of Gynaecology and Obstetrics, Shanghai Tenth People's Hospital, \\ Tongji University School of Medicine, Shanghai 200072; ${ }^{4}$ Phase I Clinical Trial Center, \\ Beijing Shijitan Hospital, Capital Medical University, Beijing 100038, P.R. China
}

Received September 11, 2013; Accepted October 30, 2013

DOI: $10.3892 /$ ijo.2014.2253

\begin{abstract}
Esophageal squamous cell carcinoma (ESCC) is one of the most common malignant tumors. Upregulation of metastasis-associated protein 1 (MTA1) has been reported to contribute to the development of esophageal squamous cell carcinoma. Therefore, the objective of our study was to identify the molecular mechanisms of MTA1 underlying the invasion and metastasis of ESCC. We overexpressed MTA1 in ESCC cells to examine the role of MTA1 in the regulation of the cell invasion. In addition, using luciferase reporter assay and electrophoretic mobility shift assays, we evaluated the binding of MTA1 to the promoter of E-cadherin. We found that MTA1 overexpression promotes invasiveness of the human esophageal carcinoma cell line EC-9706. This effect was accompanied by downregulation of the epithelial cell marker E-cadherin and upregulation of vimentin and MMP-9 luciferase reporter assays showed that MTA1 inhibited the promoter activity of E-cadherin and that this was dependent on Snail, Slug and HDAC1. We also found that Snail and Slug bound the E-boxes in the promoter of E-cadherin and recruited MTA1 and HDAC1 to suppress E-cadherin expression, as confirmed by electrophoretic mobility shift and chromatin immunoprecipitation assays. MTA1 promotes tumor invasion by downregulation of E-cadherin. These results demonstrate a novel role for MTA1 in the regulation of esophageal squamous
\end{abstract}

Correspondence to: Professor Xinghe Wang, Phase I Clinical Trial Center, Beijing Shijitan Hospital, Capital Medical University, Beijing 100038, P.R. China

E-mail: wangxh@bjsjth.cn

Dr Jin Qiu, Department of Gynaecology and Obstetrics, Shanghai Tenth People's Hospital, Tongji University School of Medicine, Shanghai 200072, P.R. China

E-mail: jeanqiuqiu@hotmail.com

*Contributed equally

Key words: esophageal squamous cell carcinoma, metastasisassociated protein 1, epithelial-to-mesenchymal transition,E-cadherin cell carcinoma invasion and provide insight into the mechanisms involved in this process.

\section{Introduction}

Esophageal squamous cell carcinoma (ESCC) is one of the most common malignant tumors in China. Similar to other malignant tumors, patients with ESCC have a poor prognosis, and this is mainly attributed to high rates of invasion and metastasis. Therefore, it is the objective of our research to identify the molecular mechanisms underlying invasion and metastasis of esophageal carcinoma. Although molecular studies on human esophageal carcinogenesis have revealed frequent genetic or epigenetic abnormalities, very little is known about the role of these alterations and the mechanisms by which they promote invasion and metastasis in this type of cancer.

One emerging group of chromatin modifiers and co-regulators is the metastasis-associated protein (MTA) family. This family is comprised of three different known genes (MTA1, MTA2 and MTA3) whose products are an integral part of the NuRD (nucleosome remodeling and histone deacetylation) complex, which is indispensable for transcriptional regulation via histone deacetylation and chromatin remodeling $(1,2)$. A large body of work has linked the upregulation of MTA1 to the maintenance and progression of more invasive phenotypes of many human cancers $(1,3,4)$. As a dual function co-regulator $(4,5)$, MTA1 operates as a transcriptional repressor of ER- $\alpha(5)$, BRCA1 (6) and p21 ${ }^{\mathrm{WAF} 11}(7)$ and as a transcriptional activator via its interaction with RNA polymerase II on the BCAS3 (8) and Pax5 (9) promoters. Moreover, recent studies have indicated for the first time that MTA1 is a genuine DNA-damage response protein, as evidenced by the induction of MTA1 by ionizing radiation and an integral component of the DNA damage response that contributes to double-strand DNA break repair (10). One mechanism by which MTA1 participates in the DNA damage response is through a stabilizing direct interaction with p53 (11).

In this study, we report that MTA1 overexpression plays a major role in the loss of E-cadherin expression in ESCC and affects the invasiveness of these cells. Our results provide 
strong evidence that MTA1 is a potent inducer of the epithelialto-mesenchymal transition (EMT) and functions as a novel repressor of E-cadherin in ESCC.

\section{Materials and methods}

Materials. ESCC cell lines were maintained in Dulbecco's modified Eagle's medium (DMEM) with $10 \%$ fetal bovine serum (FBS), $100 \mathrm{U} / \mathrm{ml}$ penicillin, and $100 \mathrm{mg} / \mathrm{ml}$ streptomycin. Total RNA was isolated from ESCC cells and primary tissues using TRIzol reagent (Invitrogen, San Diego, CA, USA) according to the manufacturer's instructions. Antibodies against Snail (ab31787), Slug (ab27568), HDAC1 (ab11966), vimentin (ab28028), MMP-9 (ab38898), E-cadherin (ab1416), MTA1 (ab751), and $\beta$-actin (ab8229) were purchased from Abcam (San Francisco, CA, USA). The MTA1 siRNA (SC35981), HDAC1 siRNA (SC44208), and Slug siRNA (SC38393) were from Santa Cruz Biotechnology (Santa Cruz, CA, USA).

Western blot analysis. Cells were harvested and lysed in $0.5 \mathrm{ml}$ lysis buffer. Lysates were clarified by centrifugation at $15,000 \mathrm{~g}$ for $10 \mathrm{~min}$. A total of $30 \mu \mathrm{g}$ of protein was processed for SDS-PAGE, which was performed using $12 \%$ gels. The proteins were electrophoretically transferred to Immobilon-P membranes (Millipore, Bedford, MA, USA). The blots were then blocked with 5\% non-fat milk in Tris-buffered saline (TBS, $\mathrm{pH}$ 7.4) for $1 \mathrm{~h}$ and then incubated with antibodies in $5 \%$ non-fat milk in TBS. After washing with TBS, the bound antibodies were visualized by enhanced chemiluminescence (Pierce Biotechnology, Rockford, IL, USA) and recorded on X-ray film (Fuji Medical, Tokyo, Japan).

Quantitative reverse transcription-PCR ( $q R T-P C R)$. Total cellular RNA was prepared using a Nucleospin RNA II kit (BD Biosciences, Franklin Lakes, NJ, USA) and cDNA was synthesized using the Superscript First-Strand Synthesis system (Invitrogen, Carlsbad, CA, USA). cDNAs were used for qRT-PCR analysis using SYBR-Green Master PCR mix on an iCycler (Bio-Rad, Hercules, CA, USA). Glyceraldehyde3-phosphate dehydrogenase (GAPDH) was included as a normalizing control. The primers used were as follows: E-cadherin, forward 5'-CGGATGACGTGTTCTACATG and reverse 5'-CGATGACGATGGGCTCGTCG; GAPDH, forward 5'-TGGTATCGTGGAAGGACTCA and reverse 5'-CCAGTAGAGGCAGGGATGAT.

Promoter reporter assay. The E-cadherin promoter sequence $(-308 /+21)$ was obtained by PCR from human genomic DNA. The PCR product was blunted, kinased, and then cloned into the pGL3basic vector (Promega, Madison, WI, USA). PCR-based site-directed mutagenesis was used for the generation of reporter gene constructs with E-box 1 and E-box 2 mutations. The mutated sequences were as follows: E-box 1, 5-gctgtggccggCCTTAGaaccetcag-3 and E-box 3, 5 -gctccgggctCGAACGgctgcagc-3. The reporter constructs were co-transfected into cells with adenovirus and siRNA. Twenty-four hours after transfection, luciferase activity was determined using a dual-luciferase reporter assay system (Promega) according to the manufacturer's instructions.
Chromatin immunoprecipitation (ChIP) assays. Briefly, cells were fixed with $1 \%$ formaldehyde, washed with cold phosphate-buffered saline, and lysed in buffer. Nuclei were sonicated to shear the DNA and the lysates were pelleted and pre-cleared. The protein-DNA complexes were incubated with protein A beads, eluted in $1 \% \mathrm{SDS} / 0.1 \mathrm{M} \mathrm{NaHCO}_{3}$ and the cross-links were reversed at $65^{\circ} \mathrm{C}$. DNA was recovered by phenol-chloroform extraction and ethanol precipitation and then subjected to semi-quantitative PCR analysis. PCR primers used for amplifying the human E-cadherin promoter were 5'-GGAGGGGTCCGCGCTGCTGA and 5'-AGCTC ACAGGTGCTTTGCAG.

Generation and infection of adenovirus expressing MTA1. An adenoviral expression system was used to express MTA1 (12). The MTA1 gene was first cloned into the pShuttle-H vector and this was then recombined with pAdEasy-1 in BJ5183 bacteria. The adenovirus was packaged and amplified in 293A cells. The adenovirus titer was determined using AdenoX rapid titer assay kit (Clontech, Tokyo, Japan). The cells were collected and plated in growth medium $24 \mathrm{~h}$ before infection. On the day of transduction, the culture medium was removed from the cells and medium containing the virus was added to the cells.

Matrigel invasion assay. Matrigel invasion assays were performed using BD BioCoat invasion chambers (BD Biosciences) and serum in complete medium served as the chemoattractant. Approximately $2.5 \times 10^{4}$ cells were added to the top chambers of 24-well transwell plates and complete media was added to the bottom chambers. After $6 \mathrm{~h}$ of incubation, the cells in the top chamber that had failed to migrate were removed and the cells that had migrated to the bottom chamber were fixed and stained.

Electrophoretic mobility shift assay (EMSA). Nuclear extracts of cells were collected using a kit from Activemotif (Tokyo, Japan). EMSAs were performed as previously reported (13). The EMSA probe sequences were as follows: WT, GCT GTG GCC GGC AGG TGA ACC CTC AGC CAA TCA GCG GTA CGG GGG GCG GTG CCT CCG GGG CTC ACC TGG CTG CAG CCA C. Mutant E1 (mE1), GCT GTG GCC GGC CTT AGA ACC CTC AGC CAA TCA GCG GTA CGG GGG GCG GTG CCT CCG GGG CTC ACC TGG CTG CAG CCA C. Mutant E3 (mE3), GCT GTG GCC GGC AGG TGA ACC CTC AGC CAA TCA GCG GTA CGG GGG GCG GTG CCT CCG GGG CTC GAA CGG CTG CAG CCA C.

Statistical analysis. Results are reported as the mean values \pm SEM. Promoter reporter and quantitative reverse transcription-PCR assays were performed in triplicate at a minimum. The data were compared using Student's t-test, and a $\mathrm{P}<0.05$ was considered statistically significant.

\section{Results}

MTA1 is a potent inducer of EMT. The epithelial-to-mesenchymal transition (EMT) is a crucial process in tumorigenesis and provides cancer cells with the ability to migrate and invade (14). The defining event for EMT is disruption of 
A

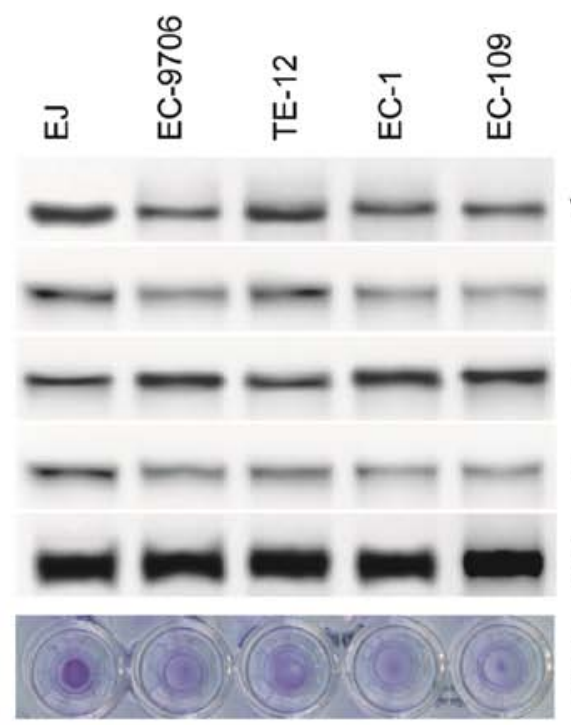

B

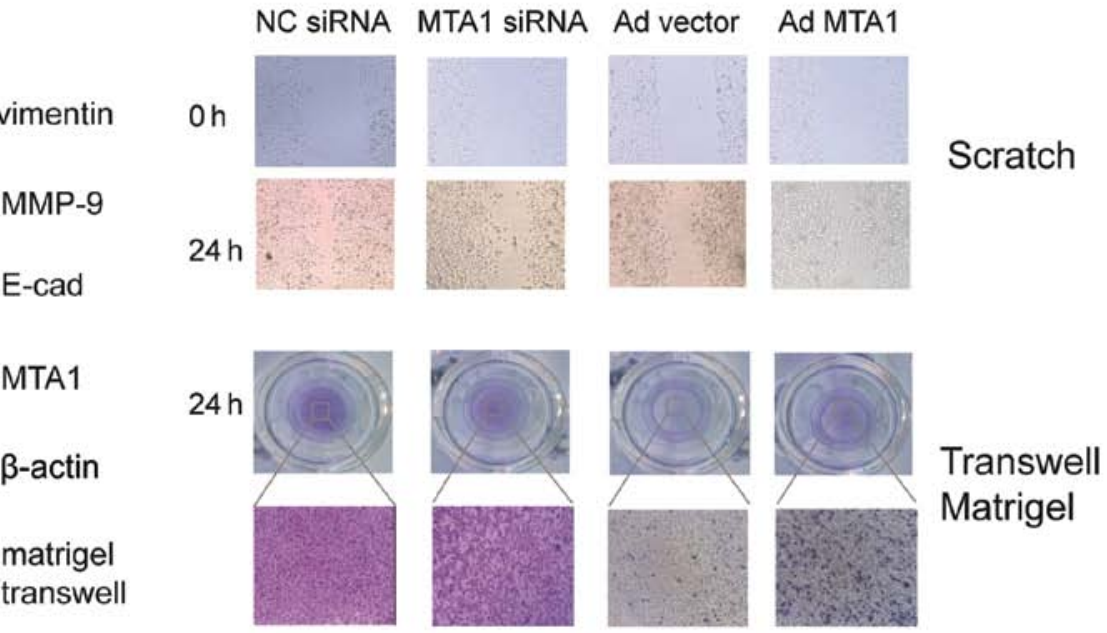

C

EJ EC-9706

NC siRNA MTA1 siRNA Ad vector Ad MTA1

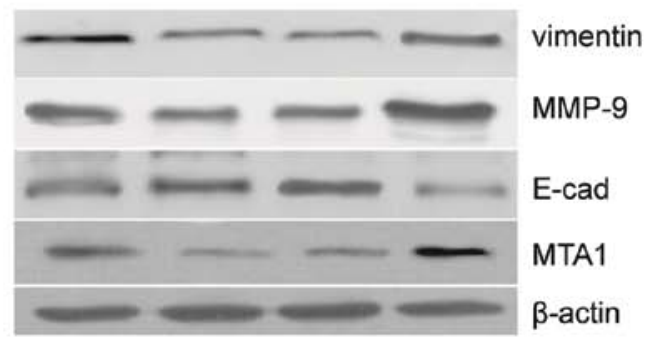

Figure 1. MTA1 promotes EMT. (A) Top, the expression of MTA1 and EMT-related proteins was detected by western blot analysis in five ESCC cell lines. Matrigel invasion assays (bottom panel). (B) Transient transfection of the MTA1 siRNA or overexpression of MTA1 in the EJ or EC-9706 cell lines significantly influenced EMT, as detected by scratch wound (top panel) and Matrigel invasion assays (bottom panel). (C) Two days after transient transfection, EMT-associated protein expression was detected by western blot analysis.

E-cadherin-mediated intercellular adhesion, which leads to decreased (Fig. 1C). Knock-down of MTA1 by siRNA resulted in the inhibition of invasion and metastasis (Fig. 1B and C). sive fibroblast-like mesenchymal phenotype. To determine whether MTA1 expression triggers EMT in esophageal cancer cells, we determined the expression of MTA1, E-cadherin (epithelial cell marker), vimentin (mesenchymal marker), and MMP-9 (cell migration marker) in esophageal cancer cell lines (EC-9706, TE-12, EC-1 and EC-109) and a bladder cancer cell line (EJ cells). Several studies have reported that EJ cells demonstrate a high invasive ability $(15,16)$. As expected, EJ cells expressed vimentin and MMP-9 at a high level and expressed lower levels of E-cadherin. Matrigel invasion assays also demonstrated the high invasion capacity of EJ cells. Compared to EJ cells, the EC-9706, TE-12, EC-1 and EC-109 cell lines had a lower capacity for invasion. We found that the expression of MTA1 was higher in EJ cells than in the esophageal cancer cells, suggesting that MTA1 expression correlated with the occurrence of EMT (Fig. 1A).

To examine the role of MTA1 in the regulation of the cell invasion, we constructed an MTA1 adenovirus expression vector (Ad-MTA1). After transfection with Ad-MTA1, EC-9706 cells (EC-9706-MTA1) demonstrated strikingly high invasiveness (Fig. 1B). As expected, the expression of vimentin and MMP-9 was increased, while that of E-cadherin was

The repression of E-cadherin promoter activity by MTA1 is dependent on Snail, Slug, and HDAC1. EMT is associated with the functional loss of E-cadherin, which is largely due to the repression of its transcription (17). An increasing number of transcription factors have been implicated in the repression of E-cadherin expression, including zinc-finger proteins of the Snail/Slug family (18-20). By using qRT-PCR assays, we found that forced expression of MTA1 in EC-9706 cells or transfection of an MTA1 siRNA into EJ cells resulted in the downregulation and upregulation of E-cadherin expression, respectively (Fig. 2A).

To further investigate the effect of MTA1 on EMT, we constructed a luciferase reporter driven by the E-cadherin promoter. The results of these experiments indicated that overexpression of MTA1 reduced the expression of E-cadherin in a dose-dependent manner (Fig. 2B). The promoter activity of E-cadherin was decreased in the presence of Ad-MTA1 alone. However, this inhibitory effect was reduced by addition of the Snail, Slug, or HDAC1 siRNAs, suggesting that MTA1 may interact with Snail, Slug, and HDAC1 to repress the transcription of E-cadherin (Fig. 2C). ChIP analysis showed an increase 
A

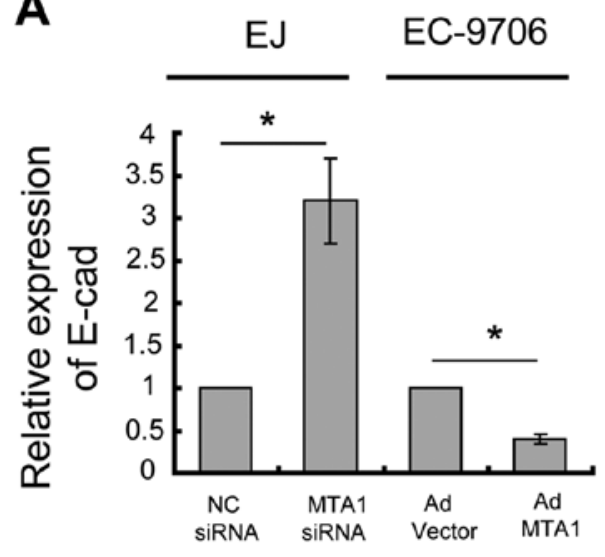

B

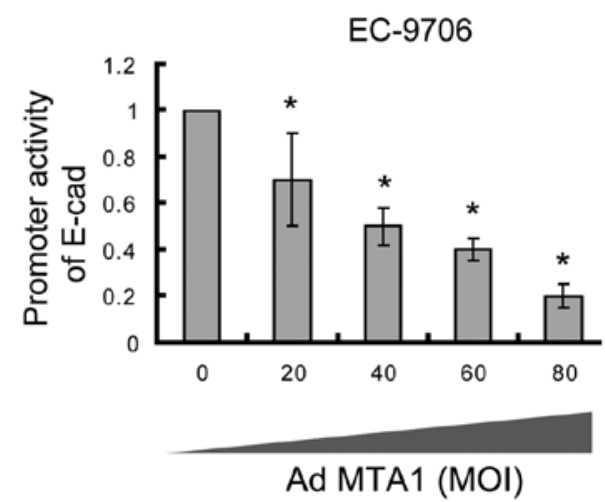

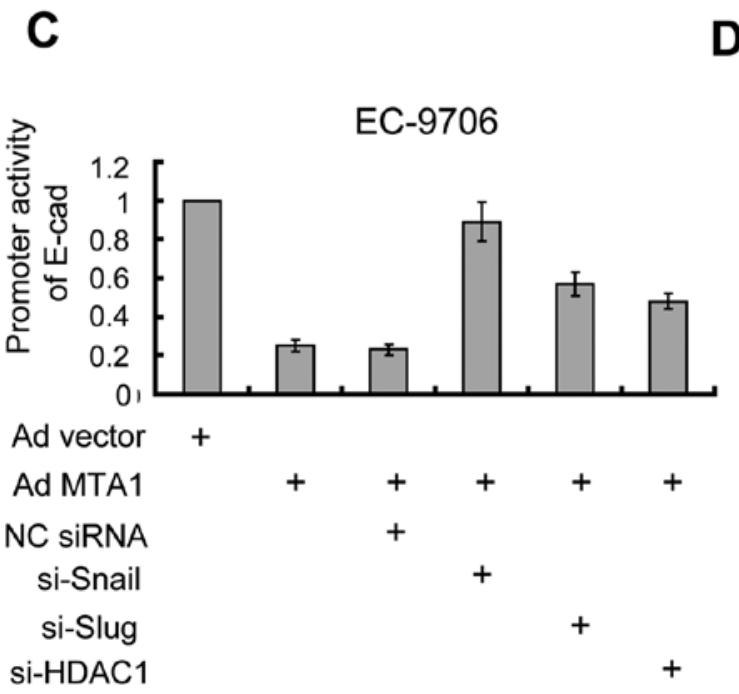

EJ

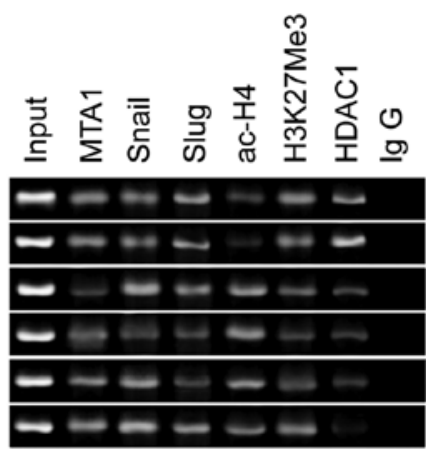

IP: E box

Figure 2. MTA1 suppression of the E-cadherin promoter is dependent on Snail, Slug, and HDAC1. (A) Knock-down or overexpression of MTA1 influenced E-cadherin expression levels, as assessed by qRT-PCR. (B) In EC-9706 cells, adenovirus expressing MTA1 (Ad-MTA1) inhibited E-cadherin promoter activity in a dose-dependent manner. (C) Mixtures of Ad-MTA1, E-cadherin reporter constructs and siRNAs were transfected into EC-9706 cells for 1 day. At the end of the culture period, cells were lysed and luciferase activity was measured. (D) Chromatin from EJ cells was harvested after the different siRNA treatments for 1 day and then precipitated with antibodies. PCR products from the ChIP assays were visualized by electrophoresis. "P<0.05; MOI, multiplicity of infection; IP, immunoprecipitation.

in the euchromatic marker tetra-acetyl-H4 and a decrease of the heterochromatin marker trimethyl-H3-K27 when the cells were treated with either the MTA1, Snail, or Slug siRNAs (Fig. 2D).

MTAl suppresses the promoter activity of E-cadherin by binding to the E1-box and E3-box sequences. It was previously shown that Snail and Slug can downregulate E-cadherin by binding to the E-box sequences in the E-cadherin promoter $(18,21)$. We proposed that MTA1 may also bind to these E-boxes. We constructed luciferase reporters containing mutations in the E1-box, E3-box, or both sequences (E1/E3; Fig. 3A). The result of these experiments showed that the inhibition conferred by MTA1 was reduced in these mutants, with the E1/E3 mutant showing the most substantial reduction (Fig. 3B). Therefore, we concluded that MTA1 may repress E-cadherin by binding, either directly or indirectly, to the E1or E3-box sequences located in the E-cadherin promoter.

To further investigate whether MTA1 bound to the E-cadherin promoter, we prepared several oligonucleotide probes, including probes to the wild-type E-box sequence, the mutated E1-box sequence, and the mutated E3-box sequence. Following incubation, DNA-protein complex interactions within E-cadherin promoter was examined. The specificity of the binding was confirmed by competition assays, as an unlabeled probe competed with the bound complex(s) in a dose-dependent manner. We found that the wild-type probe formed three complexes (designated a, b and c), but only the a or $b$ complexes were detected using the $\mathrm{mE} 1$ and $\mathrm{mE} 3$ probes, respectively. These results suggested that the E1-box and E3-box sequences participated in $b$ and a complex formation, while formation of the complex required both the E1-box and E3-box sequence (Fig. 4A). By employing supershift assays, we found that MTA1 and Snail bound to the E1-box and E3-box sequences. Although the supershift band was not observed following addition of the anti-Slug antibody, the supershift band generated using the $\mathrm{mE1}$ probe was significantly inhibited, suggesting that Slug bound to the E3-box and not to the E1-box sequence. It appears that HDAC1 does not bind to either the E1-box or the E3-box sequence, though this 
A

\begin{tabular}{|c|c|c|c|c|}
\hline \multicolumn{4}{|c|}{ E-cad Promoter } & \\
\hline Ebox 1 & & Ebox 3 & & \\
\hline CAGGTG & CAAT box & САССТG & Luciferase & E-cad WT \\
\hline-75 & & -24 & & Lead vit \\
\hline C्वसमद & CAAT box & САCCTG & Luciferase & mE-cad box1 \\
\hline CAGGT & CAAT box & CGACE & Luciferase & $\mathrm{mE}$-cad box3 \\
\hline C्वक्नव & CAAT box & CEAACG & Luciferase & mE-cad box $(1+3)$ \\
\hline
\end{tabular}

B

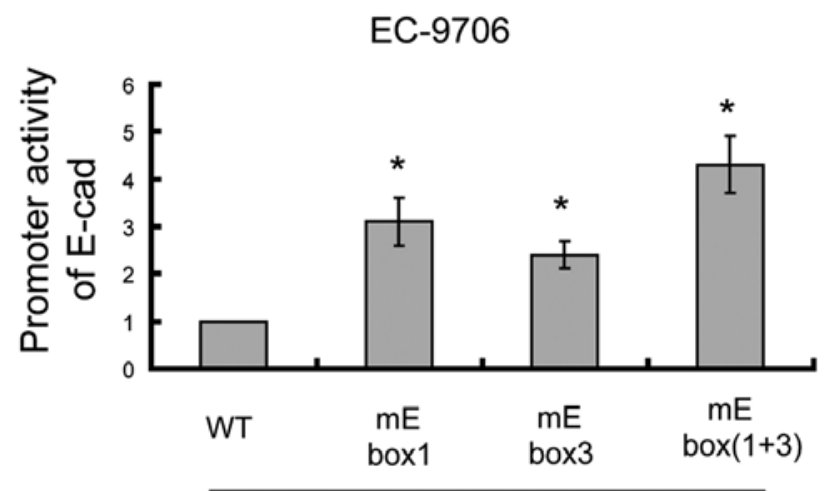

Ad MTA1

Figure 3. MTA inhibits E-cadherin expression via the E-box sequences. (A) Schematic representation of the E-cadherin reporter constructs. (B) Mutation of the E-box sequences influenced the suppressive effects of MTA1 on the promoter activity of E-cadherin. ${ }^{*} \mathrm{P}<0.05$.

A

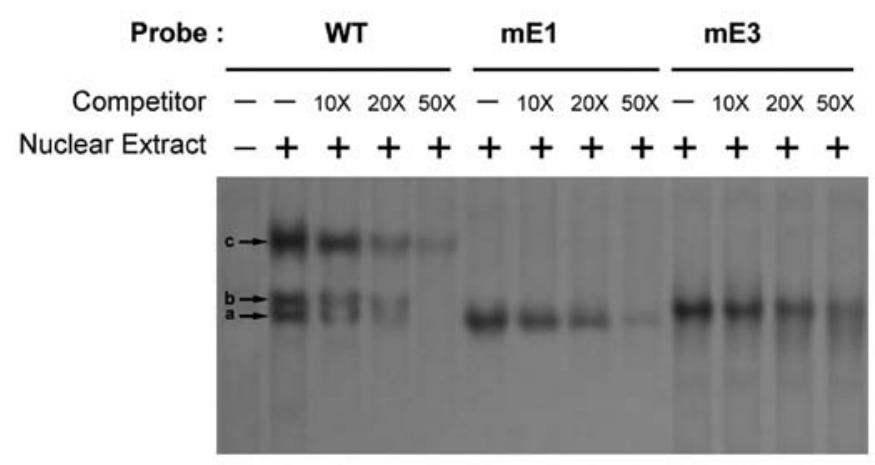

$\begin{array}{lllllllllllll}1 & 2 & 3 & 4 & 5 & 6 & 7 & 8 & 9 & 10 & 11 & 12 & 13\end{array}$

B

Probe:

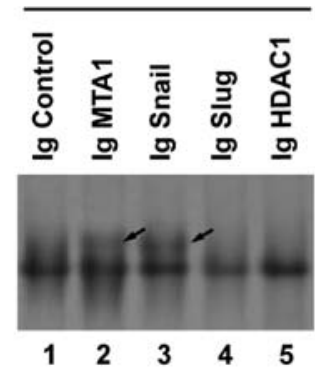

mE3

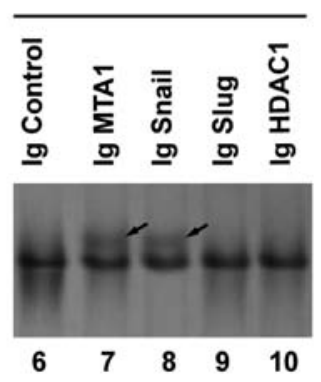

Figure 4. Binding of MTA1 to the E-cadherin core promoter bearing the two E-box sequences. (A) Upon interaction with the nuclear extract of EC-9706 cells, the probe containing the wild-type E-box sequences generated three specific bands (bands a, b, and c), which were competed with by increasing concentrations of the unlabeled oligo. Probes containing mutations of the E1-box or E3-box sequences gave rise to only one specific band. (B) Addition of the anti-MTA1 or Snail antibodies resulted in a supershifted band (indicated by an arrow). Addition of the anti-Slug antibody decreased the formation of the supershifted band.

requires further confirmation (Fig. 4B). Taken together, these findings indicated that MTA1, together with Snail or Slug, acted to repress the promoter activity of E-cadherin.

\section{Discussion}

In this study, we showed a novel role for MTA1 in the regulation of ESCC invasiveness, and this finding is likely clinically relevant. As a master dual coregulatory protein, MTA1 has indispensable transcriptional regulatory functions via its effects on histone deacetylation and chromatin remodeling. Ghanta et al compared the gene expression profiles of wildtype MTA1 and MTA1 knockout cells as well as MTA1 knockout cells re-expressing MTA1 in order to identify genuine MTA1 target genes. They found that in the presence of p53, the majority of the genes regulated by MTA1 are related to inflammatory and anti-microbial responses, whereas in the absence of p53 the predominant target genes of MTA1 are involved in cancer signaling (35). Abnormal expression or mutation of $\mathrm{p} 53$ has been reported at a high frequency in the early stages of ESCC. Thus, loss of p53 function is thought to be an important step in esophageal carcinogenesis (22-24). This suggests that in the absence of p53, MTA1 upregulation may contribute to the development of esophageal cancer. Using immunohistochemical methods, Miyatani et al (25) showed that expression of MTA1 was found in $0 \%$ of cases with normal esophageal epithelium and normal gastric epithelium, while $85.7 \%$ of cases with Barrett's epithelium and $100 \%$ of cases with gastroesophageal junction cancer were positive for MTA1 expression. Other research (26) has shown that MTA1 overexpression is detected in ESCC with no regional lymph node metastasis and that this significantly correlated with a shorter disease-free interval. Moreover, Toh et al (27) found that $42.9 \%$ of ESCCs displayed overexpression of the MTA1 protein. Also, cancers overexpressing the MTA1 protein invaded deeper into the esophageal wall and showed significantly higher degrees of lymph node metastasis, higher pathological stage, more lymphatic involvement and a poorer prognosis than the remaining cases, suggesting that MTA1 is upregulated in ESCC and contributes to its invasiveness.

EMT is a tightly controlled process that is critical for biological events such as embryomorphogenesis, development of fibrotic diseases, and tumor metastasis. EMT gives cells the ability to migrate and invade and typically reflects the plasticity of epithelial cells $(14,28,29)$. After undergoing EMT 
carcinoma cells acquire a mesenchymal phenotype, leading to enhanced motility and the ability to evade apoptosis, and these changes allow tumor cells to metastasize and establish secondary tumors at distant sites. Increasing numbers of signaling molecules have been implicated in the control of EMT-based cancer progression. Zhu et al (30) reported that EIF5A2 expression, which has a role in colorectal carcinoma (CRC) aggressiveness, was significantly correlated with tumor metastasis and short survival times. Ectopic overexpression of EIF5A2 in CRC cells enhanced cell motility and invasion in vitro and tumor metastasis in vivo as well as induction of EMT. It was shown that EIF5A2 in CRC cells substantially enhanced the enrichment of c-myc on the promoter of MTA1, suggesting that EIF5A2 plays an important oncogenic role in CRC aggressiveness via the upregulation of MTA1, thus inducing EMT. In the present study, transwell Matrigel invasion assays showed that EC-9706-MTA1 cells acquired a high level of invasiveness. A critical molecular feature of EMT is the downregulation of epithelial cell marker E-cadherin and upregulation of vimentin and MMP-9. Several studies have already shown that downregulation of MTA1 by RNAi leads to reduction in MMP-9 expression and an increase in E-cadherin expression in cancer cells $(31,32)$. This is identical to the EMT process, in which there is a decrease in the levels of E-cadherin and an increase in the levels of vimentin and MMP-9.

Snail and Slug are recognized as key inducers of EMT for their ability to repress the transcription of E-cadherin. Analysis of mouse and human E-cadherin promoters has revealed a conserved modular structure with positive regulatory elements, including two E-boxes (CACCTG) with potential roles in repression $(33,34)$. It was shown that Snail and Slug can downregulate E-cadherin by binding to the E-boxes in the promoter region $(18,21)$. As previous studies reported that MTA1 protein contained domains of many transcription factors by using structural analysis (27), our results showed that MTA1 competes with Snail, Slug and HDAC1 at the E-box sequences. MTA1 and HDAC1 form a protein complex with histone deacetylase activity that plays important roles in histone deacetylation, alteration of chromatin structure, and transcriptional control. It has been reported that the overexpression of the MTA1 protein and the acetylation level of the histone $\mathrm{H} 4$ protein are closely linked and might be useful predictors of the malignant potential of ESCC (27). Thus, we concluded that Snail, Slug, and MTA1 bind to E-boxes and subsequently recruit HDAC1 to help configure chromatin structure, leading to the silencing of E-cadherin.

In conclusion, we have determined that MTA1 is representative of a new class of transcriptional factors that function in the regulation of EMT and that play a role in the loss of E-cadherin expression in human esophageal carcinoma cells. This activity of MTA1 provides an interesting insight into the mechanisms underlying EMT-associated physiologic and pathologic processes and provides novel information useful for combating cancer metastases.

\section{Acknowledgements}

This study was supported by National Natural Science Foundation of China (81171883) to Zhi Li and National Natural Science Foundation of China (81201884) to Wenhao Weng.

\section{References}

1. Manavathi B, Singh K and Kumar R: MTA family of coregulators in nuclear receptor biology and pathology. Nucl Recept Signal 5: e010, 2007.

2. Denslow SA and Wade PA: The human Mi-2/NuRD complex and gene regulation. Oncogene 26: 5433-5438, 2007.

3. Kumar R, Wang RA and Bagheri-Yarmand R: Emerging roles of MTA family members in human cancers. Semin Oncol 30: 30-37, 2003.

4. Manavathi B and Kumar R: Metastasis tumor antigens, an emerging family of multifaceted master coregulators. J Biol Chem 282: 1529-1533, 2007.

5. Mazumdar A, Wang RA, Mishra SK, Adam L, BagheriYarmand R, Mandal M, et al: Transcriptional repression of oestrogen receptor by metastasis-associated protein 1 corepressor. Nat Cell Biol 3: 30-37, 2001

6. Molli PR, Singh RR, Lee SW and Kumar R: MTA1-mediated transcriptional repression of BRCA1 tumor suppressor gene. Oncogene 27: 1971-1980, 2008

7. Li DQ, Pakala SB, Reddy SD, Ohshiro K, Peng SH, Lian Y, et al: Revelation of p53-independent function of MTA1 in DNA damage response via modulation of the p21 WAF1-proliferating cell nuclear antigen pathway. J Biol Chem 285: 10044-10052, 2010.

8. Gururaj AE, Singh RR, Rayala SK, Holm C, den Hollander P, Zhang H, et al: MTA1, a transcriptional activator of breast cancer amplified sequence 3. Proc Natl Acad Sci USA 103: 6670-6675, 2006.

9. Balasenthil S, Gururaj AE, Talukder AH, Bagheri-Yarmand R, Arrington T, Haas BJ, et al: Identification of Pax5 as a target of MTA1 in B-cell lymphomas. Cancer Res 67: 7132-7138, 2007.

10. Li DQ, Ohshiro K, Reddy SD, Pakala SB, Lee MH, Zhang Y, et al: E3 ubiquitin ligase COP1 regulates the stability and functions of MTA1. Proc Natl Acad Sci USA 106: 17493-17498, 2009.

11. Li DQ, Divijendra Natha Reddy S, Pakala SB, Wu X, Zhang Y, Rayala SK, et al: MTA1 coregulator regulates p53 stability and function. J Biol Chem 284: 34545-34552, 2009.

12. He TC, Zhou S, Costa LT da, Yu J, Kinzler KW and Vogelstein B: A simplified system for generating recombinant adenoviruses. Proc Natl Acad Sci USA 95: 2509-2514, 1998.

13. Sun F, Xie Q, Ma J, Yang S, Chen Q and Hong A: Nuclear factor Y is required for basal activation and chromatin accessibility of fibroblast growth factor receptor 2 promoter in osteoblast-like cells. J Biol Chem 284: 3136-3147, 2009.

14. Thiery JP and Sleeman JP: Complex networks orchestrate epithelial-mesenchymal transitions. Nat Rev Mol Cell Biol 7: 131-142, 2006.

15. Yang D, Wang $\mathrm{H}$, Wang J, Zhang $\mathrm{C}$ and $\mathrm{Xu} \mathrm{H}$ : Establishment of a fluorescent implantation metastasis model of bladder cancer and real-time microscopic detection in nude mice. Asian Pac J Cancer Prev 12: 393-396, 2011.

16. Hudson MA and McReynold LM: Urokinase (u-PA) and the u-PA receptor. Modulation of in vitro invasiveness of human bladder cancer cell lines. Adv Exp Med Biol 462: 399-412, 1999.

17. Peinado H, Portillo F and Cano A: Transcriptional regulation of cadherins during development and carcinogenesis. Int J Dev Biol 48: 365-375, 2004

18. Batlle E, Sancho E, Franci C, Dominguez D, Monfar M, Baulida J, et al: The transcription factor snail is a repressor of E-cadherin gene expression in epithelial tumour cells. Nat Cell Biol 2: 84-89, 2000.

19. Guaita S, Puig I, Franci C, Garrido M, Dominguez D, Batlle E, et al: Snail induction of epithelial to mesenchymal transition in tumor cells is accompanied by MUC1 repression and ZEB1 expression. J Biol Chem 277: 39209-39216, 2002.

20. Cano A, Perez-Moreno MA, Rodrigo I, Locascio A, Blanco MJ, del Barrio MG, et al: The transcription factor snail controls epithelial-mesenchymal transitions by repressing E-cadherin expression. Nat Cell Biol 2: 76-83, 2000.

21. Prindull G and Zipori D: Environmental guidance of normal and tumor cell plasticity: epithelial mesenchymal transitions as a paradigm. Blood 103: 2892-2899, 2004.

22. Egashira A, Morita M, Yoshida R, Saeki H, Oki E, Sadanaga N, et al: Loss of p53 in esophageal squamous cell carcinoma and the correlation with survival: analyses of gene mutations, protein expression, and loss of heterozygosity in Japanese patients. J Surg Oncol 104: 169-175, 2011. 
23. Fichter CD, Herz C, Munch C, Opitz OG, Werner $M$ and Lassmann S: Occurrence of multipolar mitoses and association with Aurora-A/-B kinases and p53 mutations in aneuploid esophageal carcinoma cells. BMC Cell Biol 12: 13, 2011.

24. Yokoyama A, Tanaka Y, Yokoyama T, Mizukami T, Matsui T, Maruyama K, et al: p53 protein accumulation, iodine-unstained lesions, and alcohol dehydrogenase-1B and aldehyde dehydrogenase-2 genotypes in Japanese alcoholic men with esophageal dysplasia. Cancer Lett 308: 112-117, 2011.

25. Miyatani T, Kurita N, Mikami C, Kashihara H, Higashijima J, Yoshikawa K, et al: Malignant potential of Barrett's esophagus: special reference to HDAC-1 and MTA-1 expression. Hepatogastroenterology 58: 472-476, 2011.

26. Li SH, Wang Z and Liu XY: Metastasis-associated protein 1 (MTA1) overexpression is closely associated with shorter disease-free interval after complete resection of histologically node-negative esophageal cancer. World J Surg 33: 1876-1881, 2009.

27. Toh Y, Ohga T, Endo K, Adachi E, Kusumoto H, Haraguchi M et al: Expression of the metastasis-associated MTA1 protein and its relationship to deacetylation of the histone $\mathrm{H} 4$ in esophageal squamous cell carcinomas. Int J Cancer 110: 362-367, 2004.

28. Lee JM, Dedhar S, Kalluri R and Thompson EW: The epithelialmesenchymal transition: new insights in signaling, development, and disease. J Cell Biol 172: 973-981, 2006.

29. Voulgari A and Pintzas A: Epithelial-mesenchymal transition in cancer metastasis: mechanisms, markers and strategies to overcome drug resistance in the clinic. Biochim Biophys Acta 1796: 75-90, 2009.
30. Zhu W, Cai MY, Tong ZT, Dong SS, Mai SJ, Liao YJ, et al: Overexpression of EIF5A2 promotes colorectal carcinoma cell aggressiveness by upregulating MTA1 through C-myc to induce epithelial-mesenchymal transition. Gut 61: 562-575, 2012.

31. Jiang $Q$, Zhang $H$ and Zhang P: ShRNA-mediated gene silencing of MTA1 influenced on protein expression of ER alpha, MMP-9, CyclinD1 and invasiveness, proliferation in breast cancer cell lines MDA-MB-231 and MCF-7 in vitro. J Exp Clin Cancer Res 30: 60, 2011

32. Rao Y, Wang H, Fan L and Chen G: Silencing MTA1 by RNAi reverses adhesion, migration and invasiveness of cervical cancer cells $(\mathrm{SiHa})$ via altered expression of $\mathrm{p} 53$, and E-cadherin/betacatenin complex. J Huazhong Univ Sci Technolog Med Sci 31: $1-9,2011$

33. Behrens J, Lowrick O, Klein-Hitpass L and Birchmeier W: The E-cadherin promoter: functional analysis of a GC-rich region and an epithelial cell-specific palindromic regulatory element. Proc Natl Acad Sci USA 88: 11495-11499, 1991.

34. Giroldi LA, Bringuier PP, Weijert M de, Jansen C, van Bokhoven A and Schalken JA: Role of E boxes in the repression of E-cadherin expression. Biochem Biophys Res Commun 241: 453-458, 1997.

35. Ghanta KS, Li DQ, Eswaran J, et al: Gene profiling of MTA1 identifies novel gene targets and functions. PLoS One 6: e17135, 2011. 